\title{
Near ML detection using Dijkstra's algorithm with bounded list size over MIMO channels
}

\author{
Atsushi OKAWADO, Ryutaroh MATSUMOTO \\ and Tomohiko UYEMATSU \\ Dept. of Communications and Integrated Systems \\ Tokyo Institute of Technology, 152-8550 Japan
}

February 13, 2008

\begin{abstract}
We propose Dijkstra's algorithm with bounded list size after QR decomposition for decreasing the computational complexity of near maximumlikelihood (ML) detection of signals over multipleinput-multiple-output (MIMO) channels. After that, we compare the performances of proposed algorithm, QR decomposition M-algorithm (QRD-MLD), and its improvement. When the list size is set to achieve the almost same symbol error rate (SER) as the QRD-MLD, the proposed algorithm has smaller average computational complexity.
\end{abstract}

\section{Introduction}

The channel capacity of multiple-input-multipleoutput (MIMO) channels linearly increases with the number of antennas [1,2. Maximum-likelihood (ML) detection provides the minimum error rate. However, the computational complexity of the simple ML detection algorithm grows exponentially with the number of transmit antennas. Thus, we need an efficient algorithm that achieves similar error rate to the ML detection. The $\mathrm{QR}$ decomposition M-algorithm (QRD-MLD) [5, 6] and sphere decoding (SD) [3] are possibly the most promising algorithms. In [10, to reduce the computational complexity, Dijkstra's algorithm is applied to SD which achieves same error rate as ML detection. Both the QRD-MLD and Di- jkstra's algorithm are tree search based algorithms. Dijkstra's algorithm uses the list of unlimited size to keep detection candidates. However, the computational complexities of the QRD-MLD and Dijkstra's algorithm are still high. To reduce the computational complexity, we propose Dijkstra's algorithm with bounded list size. When proposed algorithm's list size is set to achieve the almost same symbol error rate (SER) as the QRD-MLD, the computational complexity of proposed algorithm is lower than the QRD-MLD.

This paper is organized as follows. In Section 2, we introduce the system model of MIMO channels. In Section 3, we review the QRD-MLD and its improvement, then propose Dijkstra's algorithm with bounded list size. In Section 4, we show the comparison between the computational complexity of the QRD-MLDs and proposed algorithm by computer simulations. Finally, we give the conclusion in section 5 .

\section{System model}

We consider the uncoded system with $t$ transmit antennas and $r$ receive antennas, and we assume $r \geq t$. We assume that the noise at each receive antenna is the additive white Gaussian noise (AWGN). Let $\mathrm{x}$ be a $t \times 1$ vector consisting of complex envelopes of transmitted signals with the signal constellation S, H an $r \times t$ fading matrix whose $(k, j)$ entry is a complex 
fading coefficient between $j$-th transmit antenna and $k$-th receive antenna, $\mathrm{z}$ an $r \times 1$ complex vector whose component is noise at each receive antenna, and y an $r \times 1$ complex vector whose component is the received signal component at each receive antenna. The model of this channel is written as

$$
\mathrm{y}=\mathrm{Hx}+\mathrm{z} .
$$

We assume that the receiver knows the channel state information $\mathrm{H}$ perfectly.

In this case, the ML detection of the transmitted signal over the channel (1) can be formulated as finding

$$
\hat{\mathbf{x}}_{m l}=\arg \min _{\mathrm{x} \in \mathrm{S}^{t}}\|\mathrm{y}-\mathrm{Hx}\|^{2} .
$$

\section{Near ML detection algorithm}

In this section, we propose the new near ML detection algorithm. First, to calculate (2) efficiently, we explain how to find the ML signal by tree search algorithm in Section 3.1. Then, we review near ML detection algorithms called QRD-MLD [5, 6] and its improvement [8] in Section 3.2. Finally, we propose Dijkstra's algorithm with bounded list size in Section 3.3 .

\subsection{QR decomposition}

To calculate (2) efficiently, we compute a QR decomposition of $\mathrm{H}$ and obtain an upper triangular matrix $\mathrm{R}$ and a unitary matrix $\mathrm{Q}$ with $\mathrm{H}=\mathrm{QR}$. Since $\mathrm{Q}$ is unitary,

$$
\|\mathrm{y}-\mathrm{Hx}\|^{2}=\left\|\mathrm{Q}^{*} \mathrm{y}-\mathrm{Q}^{*} \mathrm{Hx}\right\|^{2}=\left\|\mathrm{Q}^{*} \mathrm{y}-\mathrm{Rx}\right\|^{2} .
$$

Let $\xi=\mathrm{Q}^{*} \mathrm{y}=\left(\xi_{1}, \cdots, \xi_{r}\right)^{T}$. The ML detection problem (2) can be reformulated as finding

$$
\begin{aligned}
\hat{\mathbf{x}}_{m l} & =\arg \min _{\mathbf{x} \in \mathrm{S}^{t}}\|\xi-\mathrm{Rx}\|^{2} \\
& =\arg \min _{\mathbf{x} \in \mathrm{S}^{t}}\left\{\sum_{j=1}^{t}\left|\xi_{j}-\sum_{i=j}^{t} \mathrm{R}_{j, i} x_{i}\right|^{2}+\sum_{k=t}^{r}\left|\xi_{k}\right|^{2}\right\} \\
& =\arg \min _{\mathbf{x} \in \mathbf{S}^{t}}\left\{\sum_{j=1}^{t}\left|\xi_{j}-\sum_{i=j}^{t} \mathrm{R}_{j, i} x_{i}\right|^{2}\right\}
\end{aligned}
$$

The second equality above follows as the second term in the second equation is irrelevant to $\mathrm{x}$.

To calculate (4) efficiently, we consider a weighted directed graph as follows. The decisions on $x_{i}$ construct a tree where nodes at $k$-th depth are correspond to the candidate of $x_{t-k+1}$ [4, and the root node is placed at depth 0 . Then, the metric value, which is the weight of branch, between a node $\hat{x}_{i}$ that has $\hat{x}_{t}, \cdots, \hat{x}_{i+1}\left(\hat{x}_{k} \in \mathrm{S}, i+1 \leq k \leq t\right)$ as ancestor nodes from the root node to its parent node is defined by

$$
m_{i}=\left|\xi_{i}-R_{i, i} \hat{x}_{i}-\sum_{j=i+1}^{t} R_{i, j} \hat{x}_{j}\right|^{2} .
$$

The distance of each node from the root node, which is called the accumulated metric value in this paper, is equal to the sum of the metric values of branches from the root node to the node itself. The accumulated metric value from the root node to the bottom node whose depth is $t$ is

$$
\sum_{i=1}^{t} m_{i}=\sum_{j=1}^{t}\left|\xi_{j}-\sum_{i=j}^{t} \mathrm{R}_{j, i} \hat{x}_{i}\right|^{2}
$$

Because $\hat{\mathrm{x}}$ that makes (5) minimum is equal to $\hat{\mathrm{x}}_{m l}$ of (4), the shortest path from the root node to the bottom node corresponds to the ML signal [4].

\subsection{QRD-MLD}

The QRD-MLD [5, 6], which is a breadth-first tree search based algorithm, finds a near ML signal. The QRD-MLD keeps only $M$ nodes at each depth with the smallest accumulated metric values [7, instead of testing all the candidate in $\mathrm{S}^{t}$ according to (4). At each depth, only $M$ nodes make their child nodes. We call a node that makes its child node detection node in this paper.

An improvement to QRD-MLD proposed in [8] reduces the number of detection nodes from the original QRD-MLD. This improved QRD-MLD has threshold value at each depth. The depth $i$ 's threshold value $\Delta_{i}$ is defined by

$$
\Delta_{i}=E_{i, \min }+X \phi^{2}
$$


where $E_{i, \min }$ is the smallest accumulated metric value of the node at $i$-th depth in the nodes whose parent node is a detection node. $X$ is a fixed constant number, and $\phi^{2}$ is the noise variance. At each depth, select the nodes that have smaller accumulated metric value than threshold value $\Delta_{i}$. If the number of selected nodes is more than $M$, only $M$ nodes with smallest accumulated metric values are selected.

Note that both algorithms do not always find the ML signal. For small to medium $M$ values, the complexity is substantially lower than the simple ML detection algorithm. However, the final result is no longer guaranteed to be the ML signal.

\subsection{Proposed algorithm: Dijkstra's algorithm with bounded list size}

Dijkstra's algorithm is an efficient algorithm to find the shortest path from a point to a destination in a weighted graph 9]. Dijkstra's algorithm uses the list of unlimited size to keep candidate nodes. If we use Dijkstra's algorithm to find the shortest path from the root to one of nodes at the bottom depth, we can get the node with minimum $\|\mathrm{y}-\mathrm{H} \hat{\mathrm{x}}\|^{2}$ among all nodes at the bottom depth and it corresponds to the ML estimate 10. However, this algorithm still has high computational complexity. To reduce the computational complxity, we propose a modified version of Dijkstra's algorithm whose list keeps only $L$ nodes with the smallest accumulated metric values in the list.

We show Dijkstra's algorithm with bounded list size.

1. Create an empty list for nodes.

2. Insert all nodes at the first level into the list.

3. Select the node A having smallest accumulated metric value in the list and remove it from the list. If the depth of $\mathrm{A}$ is $t$, then output the node A and its ancestor nodes as the ML signal and finish this algorithm.

4. Insert all A's child nodes into the list.

5. Arrange the nodes in the list according to the accumulated metric value by the quick sort. If the

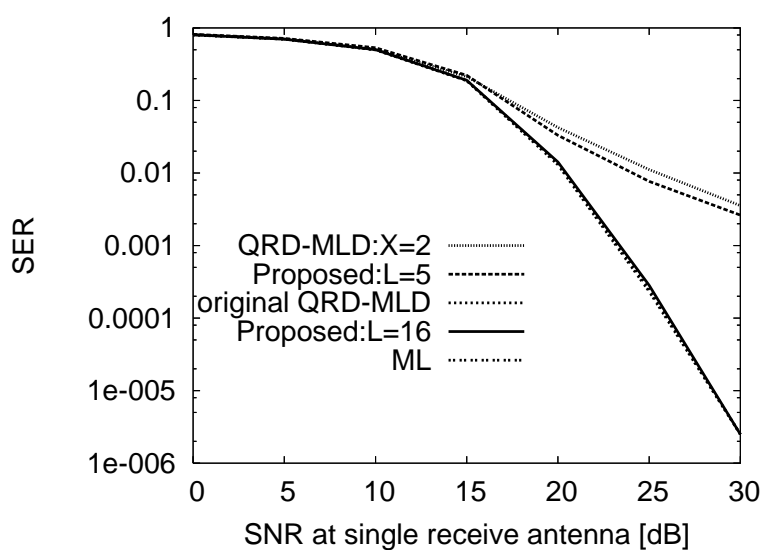

Figure 1: $(4 \times 4)$ symbol error rate

list has more than $L$ nodes, select the $L$ nodes with the smallest accumulated metric values in the list, and discard other nodes from the list.

6. Go back to Step 3.

The node whose child nodes are inserted into the list is called detection node in this paper. Because the discarded nodes, which are decided at Step 5, and their descendant nodes are not examined, the proposed algorithm dose not examine all the candidate in $\mathrm{S}^{t}$ according to (4). Thus, the proposed algorithm dose not always find the ML signal.

When we use LDPC codes [12] or turbo codes [13] after detection, we have to compute $N$ most likely signals 11. Such signals can be computed by this algorithm's modification that is finished after output $N$ signals with the smallest accumulated metric value.

\section{Computer simulation}

In this section, we compare the computational complexity, the number of detection nodes and the number of comparisons of real numbers among the proposed algorithm and the QRD-MLDs. Throughout the simulations, we consider the following system model. 


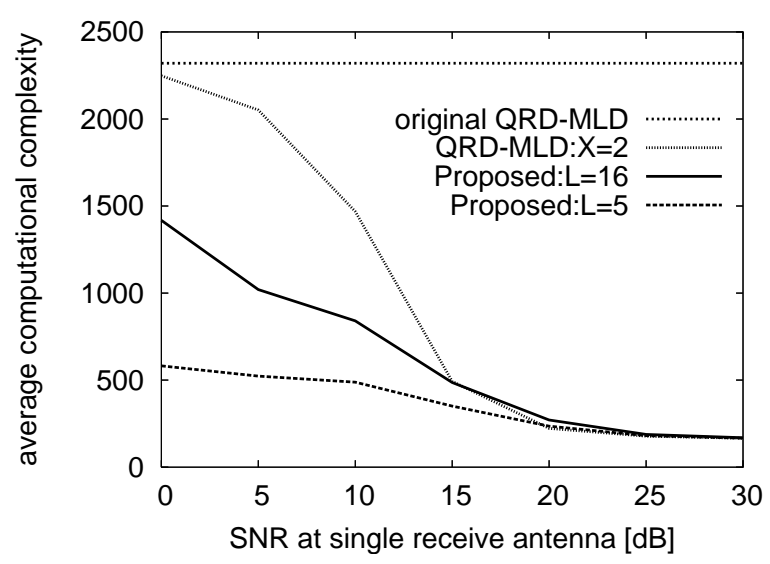

Figure 2: $(4 \times 4)$ average computational complexity

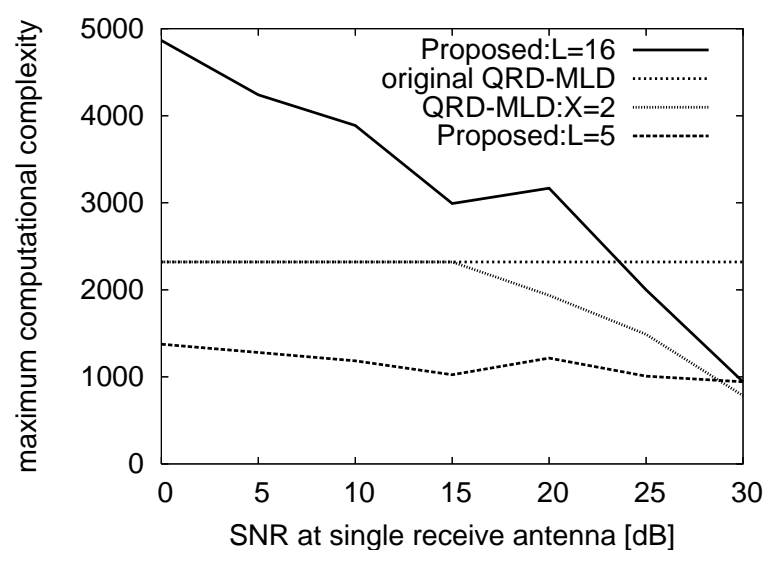

Figure 3: $(4 \times 4)$ maximum computational complexity

- We do two simulations. In the first simulation, the number of transmit antennas $t=4$, and the number of receive antennas $r=4$. In the second simulation, the number of transmit antennas $t=$ 6 , and the number of receive antennas $r=6$.

- The signal constellation at each transmit antenna is 16-QAM and all signals are drawn according to the uniform i.i.d. distribution.

- The fading coefficients obey the $C N(0,1)$ distribution, and the receiver knows it perfectly.

- The noise at each recieve antenna obeys the

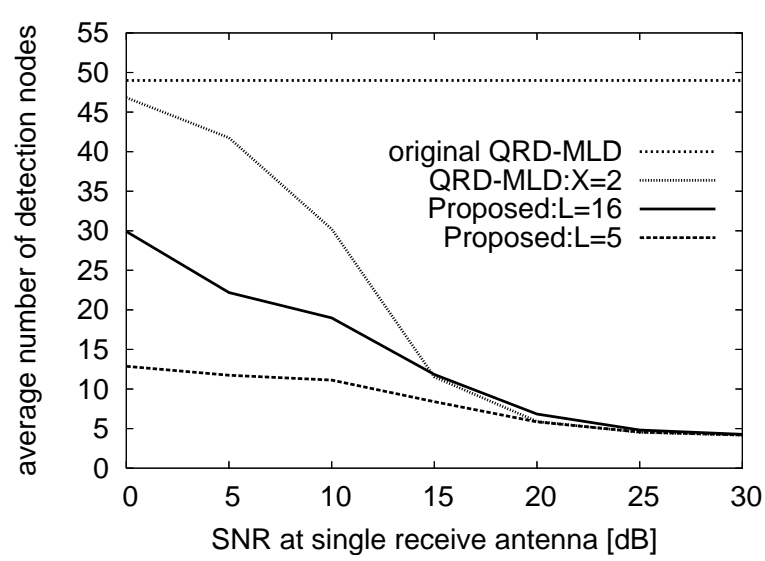

Figure 4: $(4 \times 4)$ average number of detection nodes

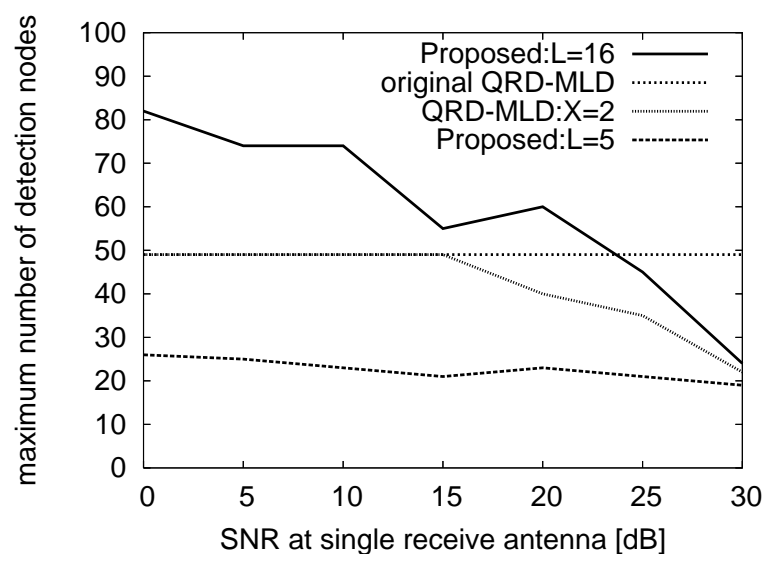

Figure 5: $(4 \times 4)$ maximum number of detection nodes

$C N(0, \phi)$ distribution. $\phi$ is caluculated by $\phi^{2}=$ $t E_{s} \times 10^{(-S N R / 10)}$, where $E_{s}$ is the average symbol energy.

- We transmit 100000 signals, which is 400000 symbols if the number of transmit antennas is 4 and 600000 symbols if the number of transmit antennas is 6 , and every 100 signals, change the fading matrix.

If $M=16$ is used and the signal constellation is 16-QAM, QRD-MLD has symbol error rate (SER) near to the ML detection [7. So, we use $M=16$. In QRD-MLD's improvement, we use $X=2$ in (6) 


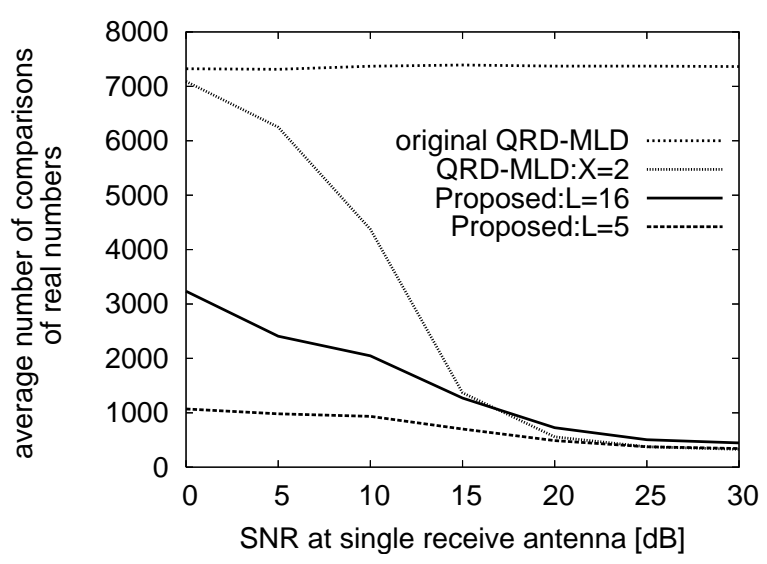

Figure 6: $(4 \times 4)$ average number of comparisons of real numbers

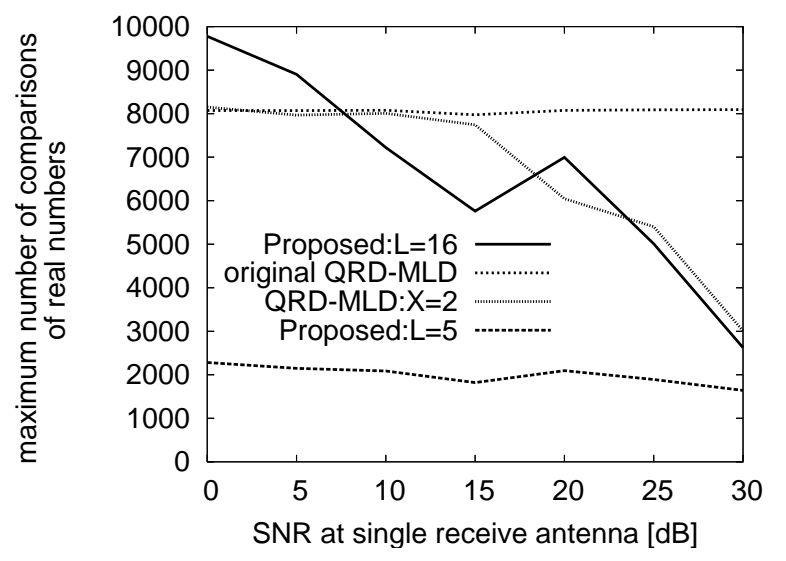

Figure 7: $(4 \times 4)$ maximum number of comparisons of real numbers

as used in [8]. In order for the proposed algorithm to have the similar SER to QRD-MLD and its improvement, we use two versions of proposed algorithm whose list sizes are $L=16$ and $L=5$. Figures 1 and 8 show that the proposed algorithm with $L=16$, the original QRD-MLD and the ML algorithm have almost the same SER throughout this simulations. The proposed algorithm with $L=5$ and QRD-MLD's improvement also have similar SER throughout this simulations.

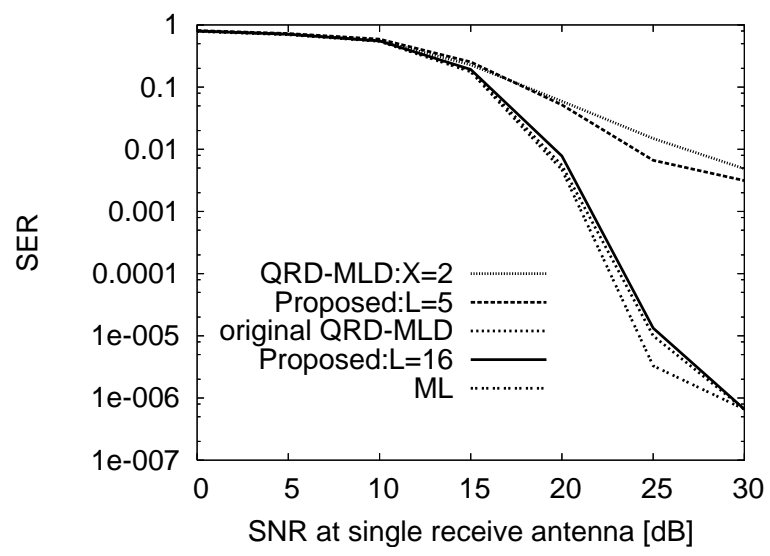

Figure 8: $(6 \times 6)$ symbol error rate

We count the number of multiplications and divisions of complex numbers as the computational complexity. Since the part of QR decomposition is the common part of all compared algorithms, we do not include that part in comparison of complexity.

In QRD-MLDs, we use the quick sort to arrange the nodes and decide $M$ nodes with the smallest accumulated metric value at each depth.

Because the QRD-MLD keeps $M$ nodes at each depth, the number of detection nodes and the computational complexity are completely determined by $M$. However, in the proposed algorithm and QRDMLD's improvement, the number of detection nodes and the computational complexity are not fixed.

Figures 1-7 are the results of first simulation whose number of transmit antennas and receive antennas are 4. Figures 8-14 are the results of second simulation whose number of transmit antennas and receive antennas are 6 .

At first, we discuss the result of first simulation. According to Figures 2, 4 and 6, the propose algorithm with $L=16$ reduece the average computational complexity, average number of detection nodes and average number of comparisons of real numbers from the original QRD-MLD. Moreover, in the case of high SNR, although the proposed algorithm with $L=16$ has much smaller SER than QRD-MLD's improvement according to Figure 1, the average computa- 


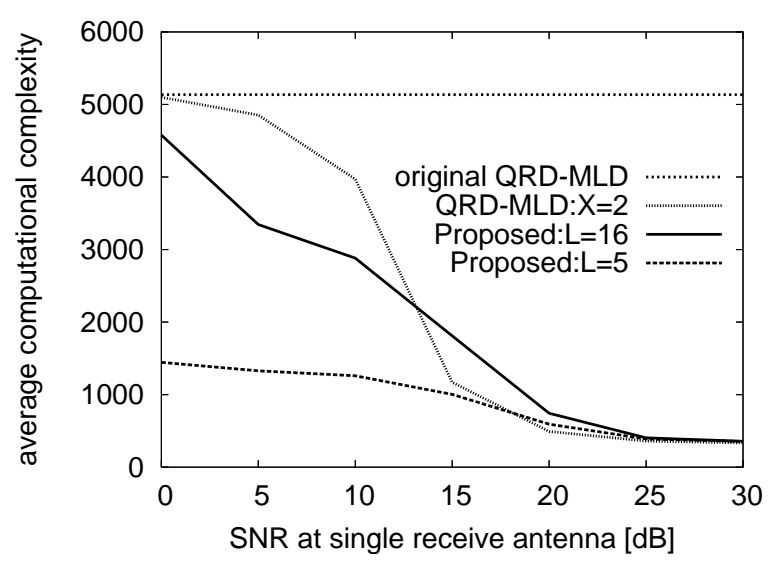

Figure 9: $(6 \times 6)$ average computational complexity

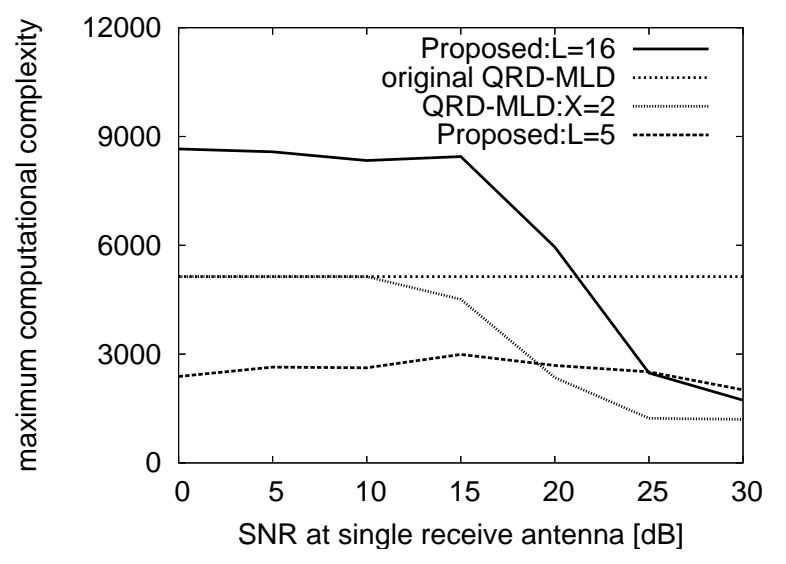

Figure 10: $(6 \times 6)$ maximum computational complexity

tional complexity of proposed algorithm with $L=16$ is almost the same as QRD-MLD's improvement. In the case of low SNR, the average computational complexity, average number of detection nodes and average number of comparisons of real numbers of the proposed algorithm with $L=5$ are lower than QRDMLD's improvement. In the case of high SNR, the average computational complexity, average number of detection nodes and average number of comparisons of real numbers of proposed algorithm with $L=5$ are almost same as QRD-MLD's improvement while the proposed algorithm has smaller SER according

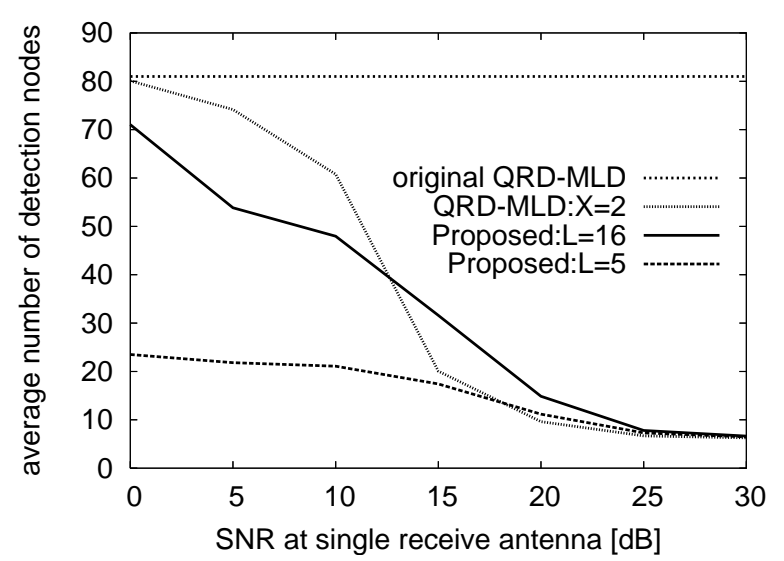

Figure 11: $(6 \times 6)$ average number of detection nodes

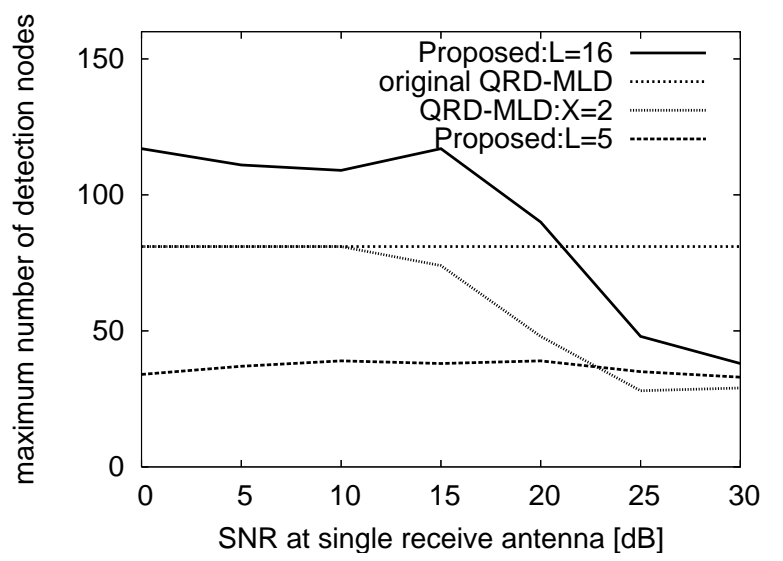

Figure 12: $(6 \times 6)$ maximum number of detection nodes

to Figure 1. According to Figures 3, 5 and 7, in the case of low SNR, maximum computational complexity, maximum number of detection nodes and the maximum number of comparisons of real numbers of the proposed algorithm with $L=16$ are higher than QRD-MLDs. However, because the average computational complexity, the average number of detection nodes and the average number of comparisons of real number of the proposed algorithm with $L=16$ are lower than QRD-MLDs, we find that the proposed algorithm rarely gets high computational complexity, large number of detection nodes or large number 


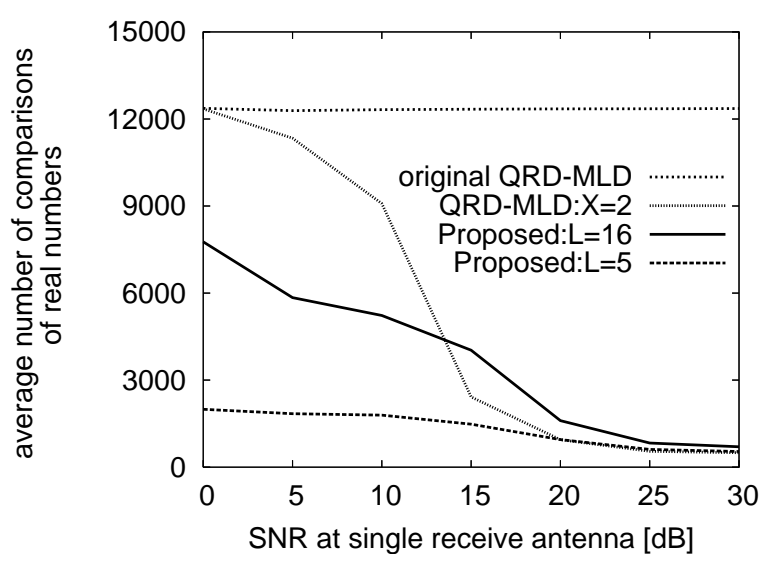

Figure 13: $(6 \times 6)$ average number of comparisons of real numbers

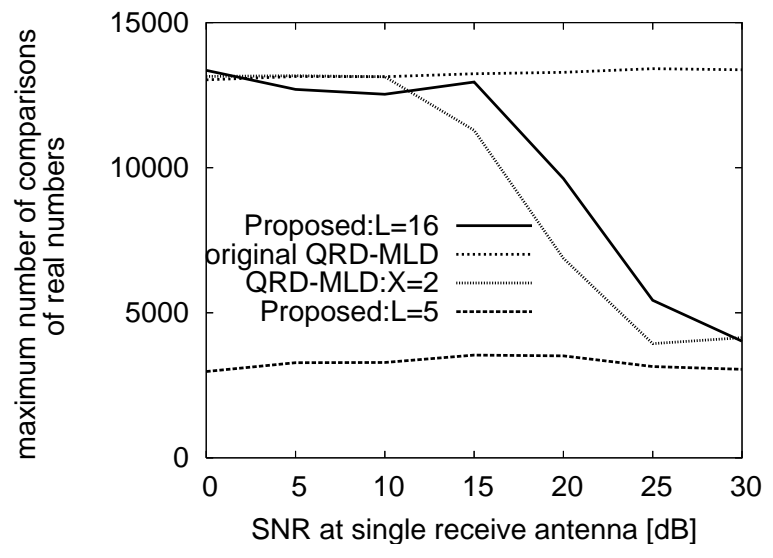

Figure 14: $(6 \times 6)$ maximum number of comparisons of real numbers

of comparisons of real numbers.

According to Figure 8-14, which is the result of second simulation, the characteristic of proposed algorithm dose not change with the number of antennas.

\section{Conclusion}

In this paper, we propose a near ML detection algorithm. When the list size is adjusted so that the proposed algorithm has the almost same symbol er- ror rate (SER) as the original QRD-MLD, the average of the computational complexity and the number of detection nodes are reduced. When the list size is adjusted so that the proposed algorithm has the almost same symbol error rate (SER) as the QRDMLD's improvement, in the case of low SNR, both the average computational complexity and average number of detection nodes are reduced and in the case of high SNR, the computational complexity and average number of detection nodes of proposed algorithm is almost same as QRD-MLD's improvement while SER of the proposed algorithm becomes smaller than QRD-MLD's improvement.

\section{Acknowledgment}

We would like to thank Prof. Kiyomichi Araki for drawing our attention to the reference [6]. This research is partly supported by the International Communications Foundation.

\section{References}

[1] E. Telatar, "Capacity of multi-antenna Gaussian channels," Europ. Trans. Telecommun., vol.10, pp.585-595, Nov. 1999.

[2] G. J. Foschini, "Layered space-time architecture for wireless communication in a fading environment when using multi-element antennas," Bell Labs Tech. J., vol.1, pp.41-59, 1996.

[3] M. O. Damen, A. Chkeif and J. C. Belfiore, "Lattice code decoder for space-time codes," IEEE Commun. Lett., vol.36, no.5, pp.166-168, Jan. 2000 .

[4] B. Hassibi and H. Vikalo, "On the spheredecoding algorithm I. Expected complexity," IEEE Trans. Signal Proc., vol.53, no.8, pp.28062818, Aug. 2005.

[5] K. J. Kim, and R. A. Iltis, "Joint detection and channel estimation algorithms for QSCDMA signals over time-varying channels," 
IEEE Trans. Commun., vol. 50, pp. 845-855, May 2002.

[6] J. Yue, K. J. Kim, G. D. Gibson, and R. A. Iltis, "Channel estimation and data detection for MIMO-OFDM systems," Proc. IEEE GLOBECOM, vol.2, pp.581-585, Dec. 2003.

[7] Y. Dai, S. Sun, and Z. Lei, " A comparative study of QRD-M detection and sphere decoding for MIMO-OFDM systems," Proc. IEEE PIMRC, vol.1, pp.186-190, Sept. 2005.

[8] H. Kawai, K. Higuchi, N. Maeda, and M. Sawahashi, "Adaptive control of surviving symbol replica candidates in QRM-MLD for OFDM MIMO multiplexing," IEEE JSAC, vol. 24, no. 6, pp.1130-1140 June 2006.

[9] A. V. Aho, J. E. Hopcoft, and J. D. Ullman, Data Structures and Algorithms, Chapter 6.3, Addison-Wesley, Reading, MA, 1983.

[10] T. Fukatani, R. Matsumoto, and T. Uyematsu, "Two methods for decreasing the computational complexity of the MIMO ML decoder," IEICE Trans. Fundamentals, vol. E87-A, no.10, pp.2571-2576, Oct. 2004.

[11] B. M. Hochwald and S. ten Brink, "Achieving near-capacity on a multiple-antenna Channel," IEEE Trans. Commun., vol. 51, vol.51, no.3, pp.389-399, Mar. 2003.

[12] R. G. Gallager, "Low density parity check codes," MIT Press, Cambridge, MA, 1963.

[13] C. Berrou and A. Glavieux, "Near optimum error correcting coding and decoding: Turbocodes," IEEE Trans. Commun., vol.44, no.10, pp.1261-1271, Oct 1996. 